\title{
Stabilization of Natural Rubber against the Effect of Cyclic heating at Different Temperature using Cashew Nut Shell Liquid (Technical CNSL)
}

\author{
Balogun Ayo Felix, Akor Joel Enemona, Isah Ilemona, Elaochi Isaac and John Godwin \\ Department of Chemistry, Kogi State College of Education (Technical), P.O.B 242 Kabba, Nigeria \\ Corresponding author: ojonugwadivine4jesus@gmail.com
}

\begin{abstract}
Natural rubber being a polymer formed in plants has very limited usefulness if not processed further. The processed (vulcanized) rubber can be further stabilized against thermal oxidation and degradation when put to use to extend the life span. The effect of cyclic heating on natural rubber treated with cashew nut shell liquid (CNSL) was determined. The natural rubber samples were dissolved in a given di-ethyl ether and a measured quantity of paraffin was added and divided into two (2) parts. The first groups were samples without stabilizing additive while the other contains stabilizing additive blended in them. The samples were heated at $60^{\circ} \mathrm{C}, 80^{\circ} \mathrm{C}$ and $100^{\circ} \mathrm{C}$ over varying periods of $3,6,9,12$ and 15 hours respectively and the compounded dried rubber was weighed into each of 12 cavities of a locally fabricate aluminium mould and the mould and its contents introduced into the oven. A solution of a measured amount of each sample in hexane was taken for viscometric measurement. Change in viscosity with length of heating shows that the various samples were degraded with time. The level of degradation of the rubber samples treated with cashew nut shell liquid (CNSL) as stabilizer showed better thermal stability compared with those without stabilizing additive.
\end{abstract}

Keywords: Natural Rubber, Cashew Nut Shell Liquid, Stabilize, Heating, viscosity

Natural rubber, a form of polymer, has been processed by vulcanization which has made it very important article of commerce and industry. Nevertheless, thermal effects on polymer degradation are a major drawback to polymeric materials. The exposure of polymers to a relatively high temperature during processing, and to moderate temperature during long term ageing, accounts for failure of most polymers. Absorption of thermal energy and atmospheric oxidation are a primary environmental factor responsible for instability of polymeric materials. However, thermal effects are generally evident in reactions that are temperature dependent which results in polymer degradation or deterioration (Lincoln, 1971). 
The resistance of polymer to thermal oxidative degradation can be increased by a variety of chemical modification. Plants sources that are processed into rubber products that are useful depends heavily on vulcanizing agents (e.g. Sulphur), accelerators (thiazoles and sulfonamides), stabilizing agents (e.g. butadiene, styrene, PVC, Aluminium hydroxide) and complex processing resins. Stabilization process against degradation in polymerization offers improved and long life span of polymers when they put long term use. Research works have shown that the level of polymer deterioration is determined by its physical structure and environmental condition of exposure. Therefore, the choice of stabilizers is directly connected to the vulnerability of the base polymer to degradation.

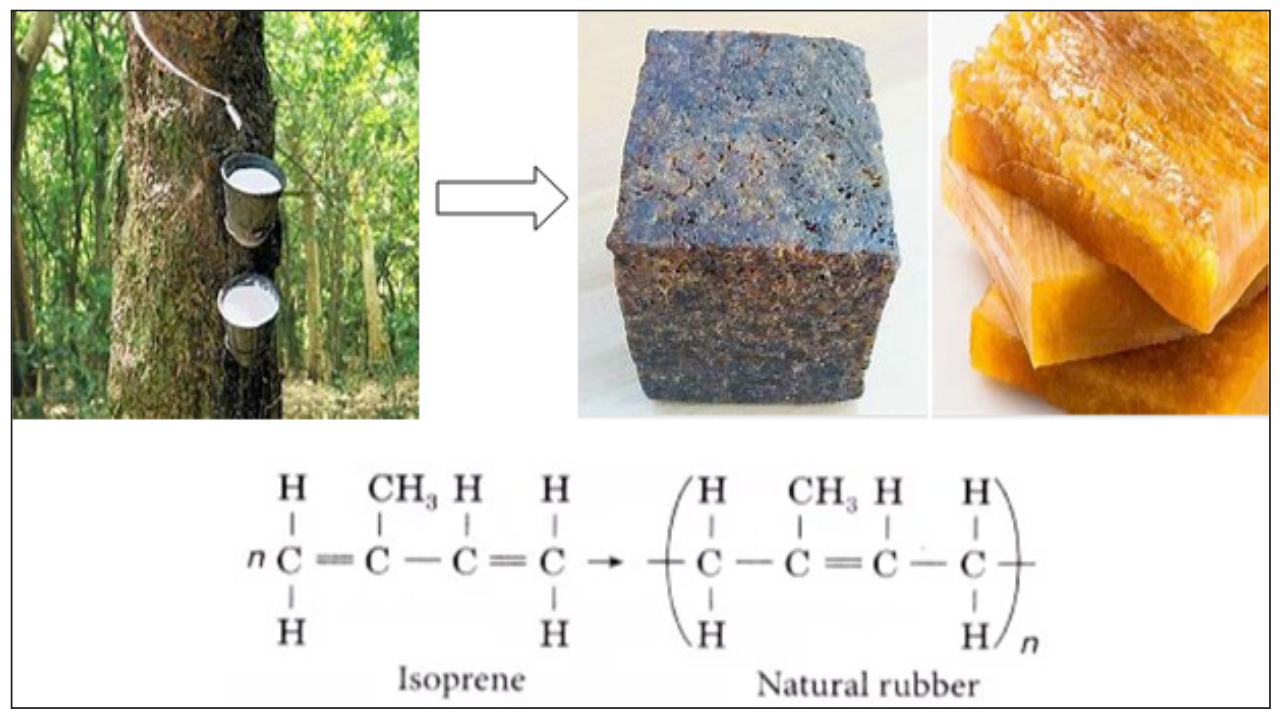

Fig. 1: Natural rubber and its chemical formula where $n=100,000$ and above

CNSL is one of the good natural sources of unsaturated long chain phenol as it is renewable and cheap substance which is easily available as by-product of industries that produces cashew (Francisco, 2006). CNSL can be applied in rubber compounding as it has many miscellaneous applications. Its low cost, availability and reactive nature makes it an alternative in industrial production of some materials. It is a by-product from cashew nut processing industries, is dark brown viscous oil present inside a soft honey comb structure of the cashew nut shell as shown in Fig. 4. It has phenolic compounds which is mainly cardanol as can be seen in Table 1. Cardanol is a monohydroxyl phenol with a long carbon chain in the meta-position as shown in Fig. 1a - d. This oil extracted from the shells contains alkyl-substituted phenolic compounds. These molecules show antioxidants properties due their structures and have the potential as substitute for phenol in phenolic-base chemical products (Damodhar et al. 2014). The use of added stabilizing agents to protect polymers against thermal oxidation is very important for most polymeric materials. It has been reported that the ageing phenomenon coincided with oxygen absorption and was retarded by phenolic substances (Semon, 1997). Eventually, a general theory of anti-oxidant emerged from the work carried out at Natural Rubber Research Producers, Research Association in England (Bateman, (2001), where it was clearly shown that hydroperoxide and free radicals were key intermediates in the oxidation of hydrocarbon. In the past decades, phenolic resins have become of increasing significance in rubber compounding. The resin based on cashew CNSL which contains phenolic bodies such as anacardic 
acid which, when treated or blended with hexamine, can be incorporated into nitrile rubber (butanieneacrylonitrile). More so, resins based on the reaction products of cardanol, phenol and formaldehyde is used to improve rubber materials resistance to cracking and ozonation degradation (Matthew et al. 2005). Various study also have shown that biological activities of the various components of CNSL have attracted considerable attention in the areas of fungicidal activity (Kubo et al. 2006), anti-inflammatory activities (Schmourlo et al. 2005), anti-oxidant activity (Amorati et al. 2001) and xanthine oxidase inhibition (Masuoka and Kubo, 2004).

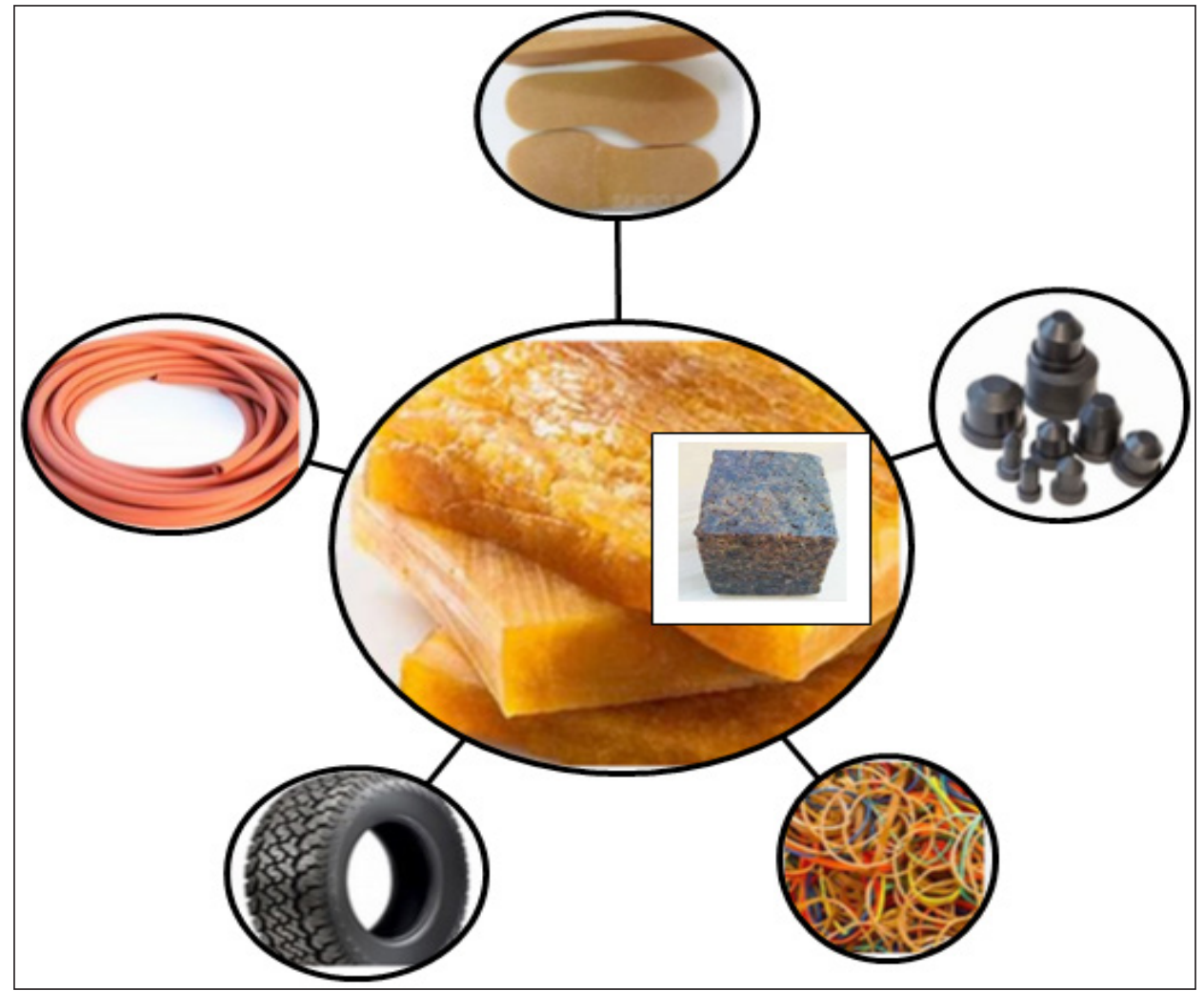

Fig. 2: Some products obtained from natural rubber that can be stabilized with CNSL against thermal degradation

Table 1: Components of solvent technical CNSL

\begin{tabular}{ll}
\hline Component & Technical extraction $\mathbf{( \% )}$ \\
\hline Anacardic acid & 0 (transformed to cardanol) \\
Cardol & $8-11$ \\
Cardanol & $83-84$ \\
2-methyl cardanol & Not documented \\
2-methyl cardol & 2 \\
Polymeric matter & 10 \\
\hline
\end{tabular}




\section{Nigeria, agriculture and diversification; a focus on cashew seed oil}

Nigeria is a producer of rubber trees (hevea brasiliensis) from which the latex is extracted (Fig. 1) and is at the verge of economic diversification due to the recent economic meltdown as a result of over dependent on the oil sector. As a matter of urgency, the agricultural sector is been exploited to reduce the pressure on the oil sector. The current global challenge due to the prevailing COVID-19 pandemic has also greatly affected the economics of many Nations with a little chances of recovery for most Nations in some years to come. This is a wakeup call to diversification of economies of all Nations at the very moment with the agricultural sector been the frontier. Nigeria is among the four major cashew producing regions including India, Brazil, and Tanzania. According to Food and Agricultural Organization (FAO), the production of cashew nuts in Nigeria in the previous decade has increased from 176,000 MT in 2000 to more than 836,500 MT currently (FAO 2016). The cashew plant has suffered neglects for a long time but, is now the focus of some industries because CNSL is the major by-product utilized by some industries especially plastic industries in which some of their products are shown in Fig. 2. There are a large number of plants materials such as trees, shrubs and herbs which are present in Nigeria that can also be used as bio-fuel. As a result of depleting reserves of fossil fuels and increasing demand for diesel, alternate sources in their availability have been receiving researchers' attention globally, Nigeria inclusive. The initiative to search for alternate innovative sources of energy to supplement or replace fossil fuels is on the rise. In recent times, rather than using fossil fuels, researchers have focused their attention upon using green methods such as plant-like seed oil and fats extracted from plants and, as a result, the most commonly used alternated fuels for now is bio-fuel (Chandrasekaran, 2016). Nearly $30-35 \%$ CNSL is available in the shell where shell percentage of nut is about $67 \%$. A report shows that a blend of $70 \%$ CNSL and $30 \%$ camphor Oil give results similar to diesel (Kasiraman et al. 2012). Another study tested commercial diesel fuel and bio-fuel from CNSL with ethanol as an additive and the result shows better performance (Velmurugan and Loganathan, 2011). Experimental Investigation of CNSL as alternative was undertaken in a work and the first trial run with a maximum of $20 \%$ CNSL-diesel blend was successfully conducted on single cylinder of CI engine (Jindal et al. 2010). Engine power output and smoke emissions were found to be satisfactory even though more testing is needed. A work was also carried out to investigate the performance and emission characteristics with $0.5 \%, 10 \%, 15 \%, 20 \%$ and $25 \%$ blends of Cardanol and Diesel. The report showed that up to $20 \%$ blends of cardinal bio-fuels may be used in CI engines without any modifications (Matthew et al. 2005).

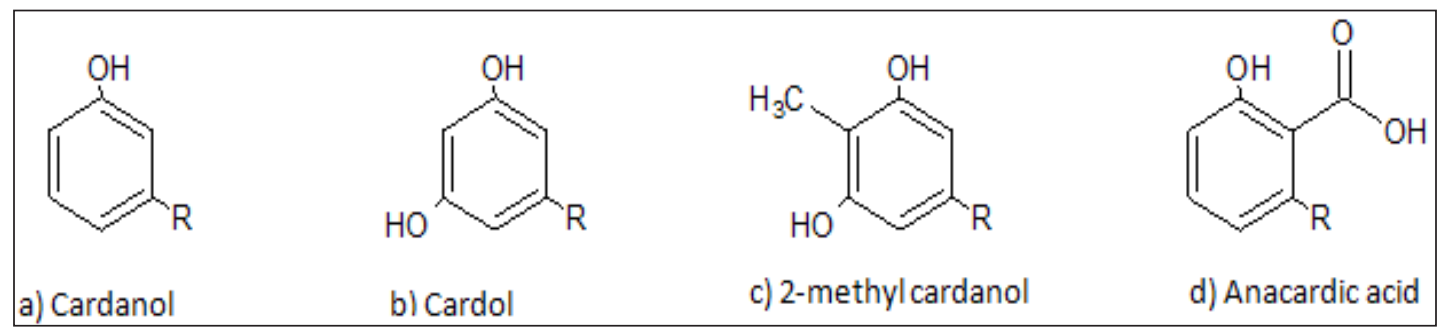

Fig. 3: Chemical constituents of technical CNSL where $\mathrm{R}$ is a long hydrocarbon

Therefore, the aim of this research work is to produce and stabilize natural rubber against the effect of cyclic heating using cashew nut shell liquid (technical CNSL) at varied temperature. This was achieved by roasting cashew nut shell liquid (technical CNSL) to obtain the liquid portion, treating natural rubber 
to obtain CSNL treated rubber, determining the thermal stability of the treated and untreated natural rubber against thermal degradation by heating at various temperature as a function of time and finally, measuring the viscosity of the rubber solution at different periods of heating.

\section{MATERIALS AND METHODS}

Analytical reagent grade chemicals and distilled water were used throughout the study. All glassware and plastic containers were washed with detergent solution followed by soaking in $20 \%(\mathrm{v} / \mathrm{v})$ nitric acid and rinsed with distilled water.

\section{Equipment}

Measuring cylinder, volumetric flask, conical flask, sieve, oven, magnetic Stirrer, digital Viscometer, locally fabricated mould with cavities and $\mathrm{pH}$ meter were used in this work.

\section{Chemicals}

Sulphur, mecapto benzyl thiazol disulphide (M.B.T.S), paraffin oil, n-Hexane, di-ethyl ether, stearic acid, zinc oxide, calcium carbonate and cashew Nut shell Liquid (technical CNSL) were used in this experiment.

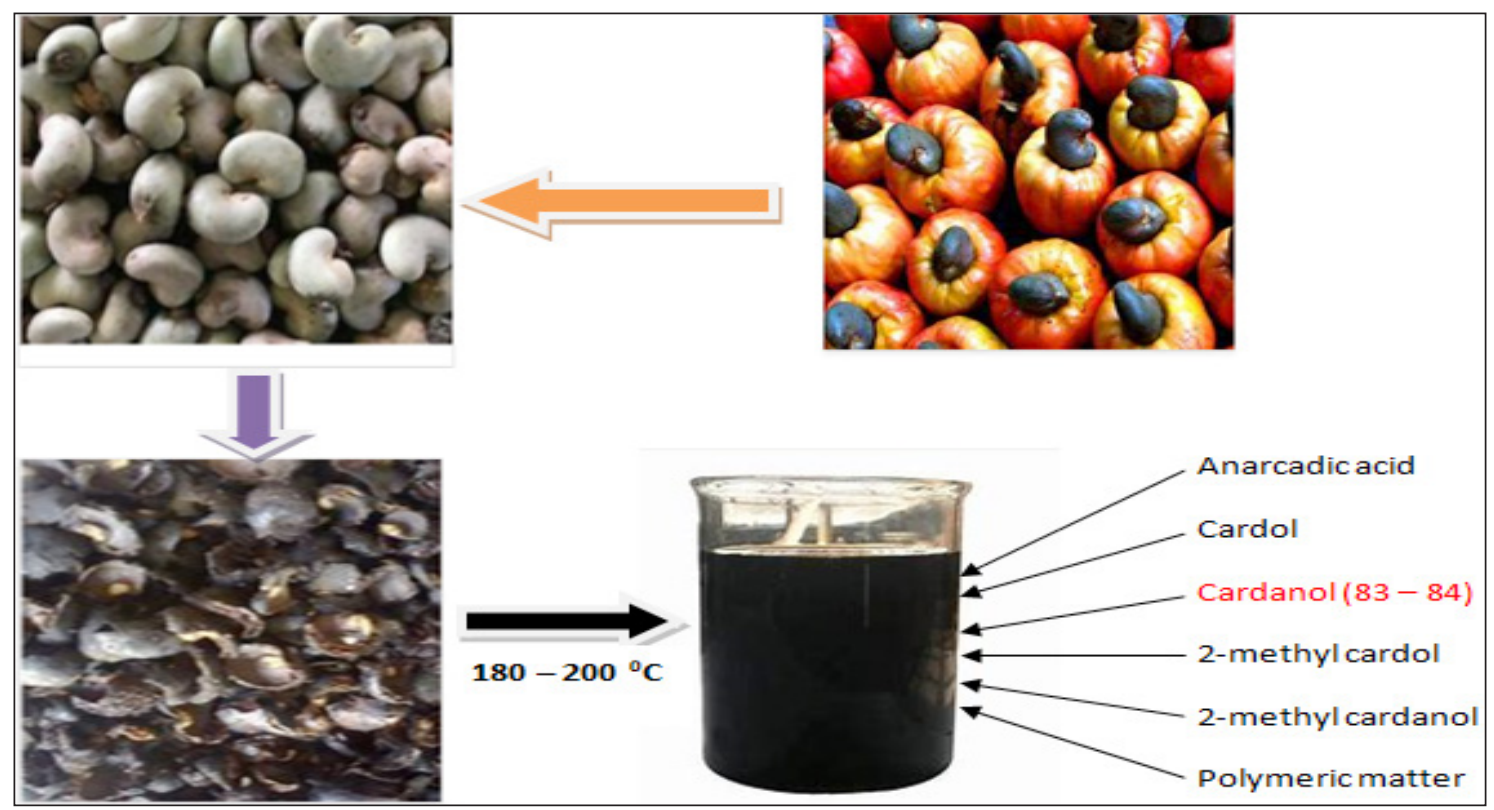

Fig. 4: Schematic diagram for preliminary processing of cashew seed to collect CNSL

\section{Sampling of Natural Rubber and Cashew Nut}

Raw Smoked Rubber was purchased from Rubber Research Institute, Benin, South South, Nigeria and taken to the laboratory for further treatment. Cashew seed was purchased from cashew farmers in Egdeda, Kabba, Kogi State and taken to the laboratory for further treatment. 


\section{Sample Treatment and Analyses}

The purchased cashew nut samples were washed thoroughly with detergent, rinsed with distilled water to ensure high degree of purity of the product and dried under the sun for one month for easy shelling. The Shell was removed using knife (plier-like) while wearing hand gloves.

\section{Procedure for Cyclic Heating}

Two procedures are followed for cyclic heating; the first step involves the blending of raw rubber with only paraffin oil while the second step involves blending of $50 \%$ solution of CNSL in paraffin with the raw rubber prior to heating.

Step 1: Typically, $4.00 \mathrm{~g}$ of raw rubber was weighed into a beaker, $16.00 \mathrm{~mL}$ of di-ethyl ether and 0.10 $\mathrm{mL}$ of paraffin was added to soften the rubber chains. After blending, the blend was allowed to dry for 12 hrs. The dried blend was placed in a $500 \mathrm{~mL}$ beaker and transferred into pre-heated oven at $60{ }^{\circ} \mathrm{C}$ for an $\mathrm{hr}$ earlier. $1.00 \mathrm{~g}$ of this sample was taken at the end of every four hrs of heating. A total of four samples were taken over a period of 15 hours of cyclic heating, representing samples that were heated for 3, 6, 9,12 and 15 hrs respectively. The experiment was also done at 80 and $100{ }^{\circ} \mathrm{C}$.

Step 2: Typically, $4.00 \mathrm{~g}$ of raw rubber was weighed into a beaker, $16.00 \mathrm{~mL}$ of di-ethyl ether and 0.10 $\mathrm{mL}$ of $25 \%$ of CNSL was added in paraffin oil to soften the rubber chains. After blending, the blend was allowed to dry for $12 \mathrm{hrs}$. The dried blend was placed in a $500 \mathrm{~mL}$ beaker and transferred into pre-heated oven at $60{ }^{\circ} \mathrm{C}$ for an hr earlier. $1.00 \mathrm{~g}$ of this sample was taken at the end of every four hrs of heating. A total of four samples will be taken over a period of $15 \mathrm{hrs}$ of cyclic heating, representing samples that are heated for 3, 6, 9, 12 and 15 hrs respectively. The experiment was also done at 80 and $100{ }^{\circ} \mathrm{C}$.

\section{Procedure for Moulding}

There are two steps involved in this stage: rubber compounding and vulcanization of the compounded rubber.

Step 1: A $50.00 \mathrm{~g}$ of rubber was weighed into a container to which $200 \mathrm{~mL}$ di-ethyl ether added and container covered up to reduce solvent vaporization. After a period of $12 \mathrm{hr}, 50.00 \mathrm{~g}$ of calcium carbonate was added and churned which followed by the addition of $2.00 \mathrm{~g}$ Zinc oxide and $4.00 \mathrm{~g}$ stearic acid which has been previously sieved and, after churning to blend, $1.00 \mathrm{~g}$ of M.B.T.S was added. After mixing with the rubber solution, $2.50 \mathrm{ml}$ of $50 \%$ solution of CNSL in paraffin was added and blended. Finally, 1.00 $\mathrm{g}$ of sieved sulphur was added and blended.

The above procedure was repeated for untreated raw rubber that had been heated in a cycle of 3, 6, 9, 12 and 15 hrs respectively.

Step 2: A $6.54 \mathrm{~g}$ of compounded dried rubber was weighed into each of cavities in an aluminium mould and the mould and its contents were introduced into oven which had been pre-set at $150{ }^{\circ} \mathrm{C}$ over a period of $1 \mathrm{hr}$. The mould and its content was left for a period of $1 \mathrm{hr}$ and 30 minutes in the oven, removed, cooled and opened to remove the cast product.

The second step was repeated for rubber samples that had been heated for 3, 6, 9, and $12 \mathrm{hrs}$ which had equally been compounded as given above. 


\section{Procedure for Viscometric Measurement}

A $1.25 \mathrm{~g}$ of cyclically heated rubber samples compounded with lipid only and dissolved in $80 \mathrm{~mL}$ n-hexane was made up to $100 \mathrm{~mL}$ in a volumetric flask. $80 \mathrm{~mL}$ of this was transferred into a beaker and taken to for viscometric measurement. The viscosity of the solution is then taken from the instrument in centipoises (cp) as shown in table....

\section{RESULTS AND DISCUSSION}

\section{Extraction of Cashew Nut Shell Liquid (CNSL)}

The CNSL in this study was obtained by roasting the shell (Thomas et al. 2017). This technical CNSL was done at the temperature between 180 to $200{ }^{\circ} \mathrm{C}$. This method recovers $85-90 \%$ of the liquid. A dark-brown liquid was obtained which research have has different percentage of components as shown in table 1 (Klimisch, 1997). The characteristics properties of the technical CNSL have been determined according to methods reported in a research work (Chaudhari et al. 2012) using standard equipment/ apparatus following standard methods and the results are represented in tables 2 and 3 for physical and chemical properties respectively. The viscosities study of the CNSL treated and untreated natural rubber as represented in Figs. 5, 6 and 7 show that the treated samples have higher viscosity values than the untreated ones. Fig. 6 show that at $60^{\circ} \mathrm{C}$ for $15 \mathrm{hrs}$ of heating, the treated samples were stable to thermal treatment while the untreated samples were relatively stable at the same temperature for up to $9 \mathrm{hrs}$ but there were a drop in viscosity after the 9 th $\mathrm{hr}$.

Table 2: Physical properties of CNSL at room temperature

\begin{tabular}{ll}
\hline Color & Dark brown \\
\hline Odor & Choke \\
Specific gravity & 0.35 \\
Boiling points & 94.0 \\
Refractive index & 1.23 \\
Density $(\mathrm{g} / \mathrm{cm} 3)$ & 0.90 \\
\hline
\end{tabular}

Table 3: Chemical properties of CNSL at room temperature

\begin{tabular}{ll}
\hline Free fatty acid & 68 \\
Acid value & 1.59 \\
Saponification value $(\mathrm{mgKOH})$ & 157 \\
Iodine value $(\mathrm{mgKOH} / \mathrm{g})$ & 86 \\
$\mathrm{Ph}$ & 5.78 \\
\hline
\end{tabular}

This suggests that the treated with CNSL has more resistance to thermal degradation compared to the untreated one. Similar results were also obtained when the temperature was increased from 60 to $80{ }^{\circ} \mathrm{C}$ with only a point drop in the viscosity values of the treated samples while the results as represented in Fig. 6 show that there was a further drop in the viscosity values for the untreated samples. Fig. 7 also show that as the temperature was further increased to $100{ }^{\circ} \mathrm{C}$, there were sharp drops in the viscosity 


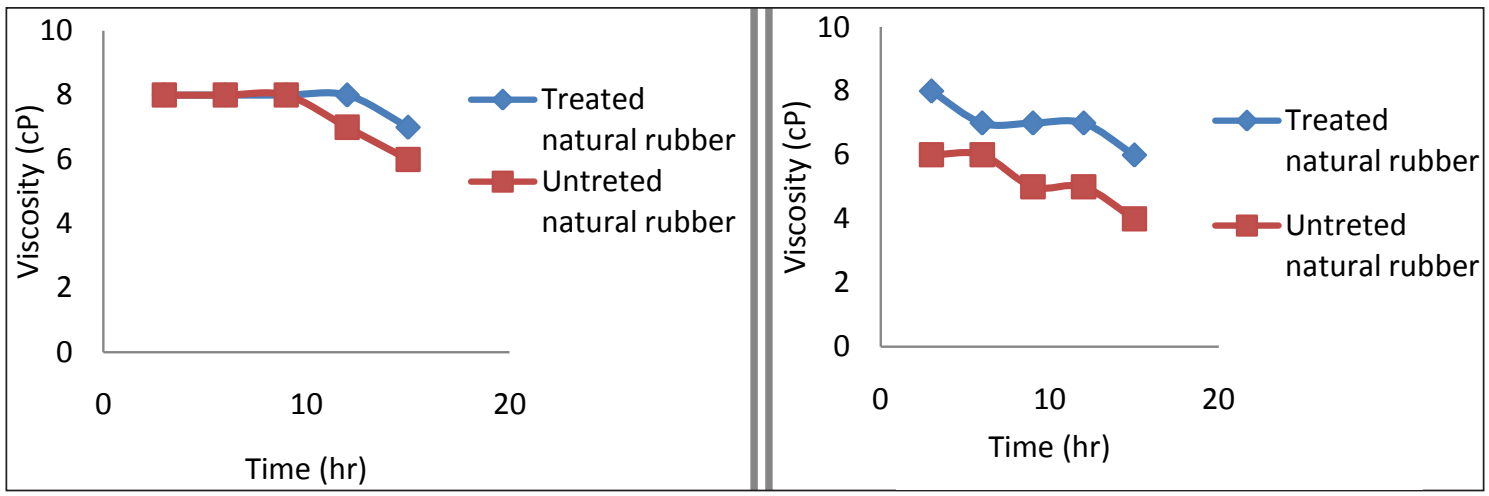

Fig. 5: Viscosities of treated natural rubber with $\mathrm{CNSL}$ and untreated rubber at $60^{\circ} \mathrm{C}$

Fig. 6: Viscosities of treated natural rubber with CNSL with untreated natural rubber at $80{ }^{\circ} \mathrm{C}$

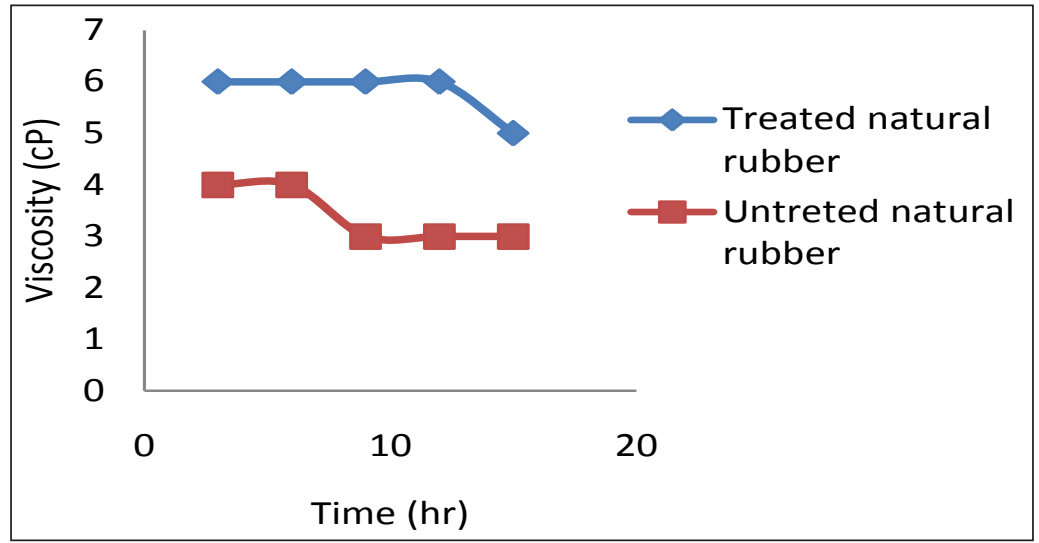

Fig. 7: Viscosities of treated natural rubber with CNSL and untreated natural rubber at $100{ }^{\circ} \mathrm{C}$

values for the untreated samples while the treated ones remained relatively stable. Generally, viscosity decrease with increase in temperature which can be seen from the results obtained in this research work. This is because at higher temperature, particles have greater thermal energy and can easily overcome the attractive forces binding molecules together (cohesive forces between molecules decreases as temperature increases). This means that as temperature increases, there is an increase in the molecular interchange as molecules move faster at higher temperatures. Therefore, the results obtained above show that the use or incorporation of CNSL as a stabilizing agent helps in the resistance of natural rubber against thermal degradation. Analysis of CNSL show that it contains high content of unsaturated long chain phenol which suggests that its chemical structure form a cross link during vulcanization of rubber to prevent molecular flow when exposed to thermal treatment. Cardanol which is the main constituent of CNSL also has an antioxidant property due to its structure and it prevents aging in rubber materials as phenolic substances have the ability to retard oxygen adsorption. Also, larger molecules have stronger intermolecular forces such as London forces which connect them to one another with greater power to inhibit molecular flow resulting in higher viscosity. This is because viscosity depends on strength of attractive forces between 
molecules as a result of composition, size and shape. Therefore, CNSL, in addition to molecular structure, also increases the molecular size and shape of natural rubber to resist molecular flow as a result of thermal treatment resulting in stability of natural rubber.

\section{CONCLUSION}

Cashew nut shell liquid (CNSL) was successfully extracted from cashew seed by roasting (technical CNSL) and the physical and chemical properties were determined. The viscosity of the technical CNSL treated natural rubber and the untreated samples were taken at different times and different temperatures for viscometric measurement. The results obtained show that the viscosities of the technical CNSL treated natural rubber at varied temperature were more stable compared to that of untreated natural rubber. Therefore, CNSL can be employed as stabilizing agent against thermal oxidation in rubber vulcanizing.

\section{REFERENCES}

1. Amorati, R., Pedulli, G.F., Valgimgli, L., Attanasi, O.A., Fillipone, P. and Fiorucci, C. 2001. Absolute Rate Constants for the Reaction of Peroxyl Radicals with Cardanol Derivatives. Journal of the Chemistry society Perkin Transactions, 2: 2142 - 2146.

2. Bateman, L. 2001. Quarterly Review, National Rubber Producers Research Association, London. 8: 147.

3. Chandrasekaran, S. 2016. Analysis of Cardinol as a Substitute for diesel. International Journal of Managing Value and Supply Chains (IJMVSC); 7(2).

4. Chaudhari A.P., Thakor, N.J., Mahale, D.M., Haldankar, P.M. and Sonawane, S.P. 2012. Physical Properties of Cashew Nut Liquid. International Journal of Application or Innovation in Engineering and Management, 1(2): $1-3$.

5. Damodhar, J.G. and Ramesh, S.B. 2014. Review on Extraction and Isolation of Cashew Nut Shell Liquid. International Journal of Innovations in Engineering Research and Technology (IJIERT); 1(1): 232 - 244.

6. FAO. 2016. Cashew production in Africa, 1961 - 2000. Food and Agriculture Organization of the United Nations. Production database, http://apps.fao.org/

7. Francisco, H.A.R., Judith, P.A.F., Nagila, M.P.S.R., Francisco, C.F. and Jose, O.B.C. 2006. Antioxidant Activity of Cashew Nut Shell Liquid (CNSL) Derivatives on Thermal Oxidation of Synthetic cis-1, 4-polyisoprene. Journal of Brazilian Chemical Society; 17(2).

8. Jindal, S., Nandwana, D., Rathore, N. and Vashistha, V. 2010. Experimental Investigation of The Effect of Compression Ratio and Injection Pressure in a Direct Injection Diesel Engine Running on Jatropha Methyl Ester. Journal of Applied Thermal Engineering, 30: 442 - 448.

9. Kasiraman. G, Nagalingam, B and Balakrishnan, N. 2012. Performance, Emission and Combustion Improvements in a Direct Injection Diesel Engine using Cashew Nut Shell Oil as Fuel with Camphor oil Blending. J. Renewable Energy, 47: 116 - 124.

10. Klimisch, H.J. 1997. A Synthetic Approach for Evaluating the Quality of Experimental Toxicology and Ecological Data. Regul. Toxicol. Pharmacol., 25: 1 - 5. 
11. Kubo, I., Masuoka, N., Ha T.J. and Tsujimoto, K. 2006. Antioxidant Activity of Anacardic Acid. Food Chemistry; 99: 555 - 562.

12. Lincoln, H.W. 1971. Polymer Stabilization. Bell tel. incorporated, Murray Hill, New Jersey; pp. 113, $161-247$, and 249.

13. Mallikappa, D., Reddy, R.P. and Murthy, C.S. 2012. Performance and Emission Characteristics of Double Cylinder CI Engine with Cardanol Biofuel Blends. Journal Renewable Energy, 38: 150 - 154.

14. Masuoka, N. and Kubo, I. 2004. Characterization of Xanthine Oxidase Inhibition by Anacardic Acid. Biochemica et Biophysica Acta., 1688: 245 - 249.

15. Matthew, O.E., Labake, F. and Rita, N.E. 2005. Extraction of Poly-phenols from Cashew Nut Shell. Journal of chemical Society; 2(12): 34 - 44.

16. Schmourlo, G., Mendoca-Filho, R.R., Alviano, C.S. and Costa, S.S. 2005. Screening of Anti-fungal Agents using Ethanol and Bioautography of Medicinal and Precipitation Food Plants. Journal of Ethnopharmacology; 15: 563 - 568.

17. Semon, W.L. 1997. History and uses Materials which Improves Ageing; the Chemistry and Technology of Rubber. David, C.C. and Blake, J.T. Edition, Reinhold, New York, pp. 414.

18. Thomas, B., Shiori, T., Ippei, N., Ebenezer, O.O. and Chul-Sa, K. 2017. Isolation and Characterization of the Insecticidal Compounds in Anacardium Occidentale (cashew nut) Shell Liquid against the Rice Weevil, Sitophilus oryzaeL. (Coleoptera: Curculionidae). Journal of Entomology and Zoology Studies; 5(2): 1241 - 1246.

19. Velmurugan, A and Loganathan, M. 2011. Performance and Emission Characteristics of a DI Diesel Engine Fuelled with Cashew Nut Shell Liquid (CNSL) - Diesel Blends. World Academy of Science, Engineering and Technology, pp. 58. 